\title{
Building Health Systems Science Education from the Core Domain of Interprofessional Education at Virginia Tech Carilion School of Medicine
}

\author{
David W. Musick ${ }^{1,2}$ (1) $\cdot$ Richard C. Vari $^{3} \cdot$ M. Suzanne Kraemer ${ }^{4}$. David B. Trinkle ${ }^{2,5} \cdot$ Patty M. Vari $^{6} \cdot$ Judy C. Smith $^{7}$. \\ Lee A Learman ${ }^{8}$
}

Accepted: 29 October 2020 / Published online: 23 November 2020

(C) International Association of Medical Science Educators 2020

\begin{abstract}
The Virginia Tech Carilion School of Medicine (VTCSOM) is a 4-year allopathic medical school in Roanoke, VA. The curriculum is organized into four learning domains: basic science, clinical science, research, and interprofessionalism (IPE). A recent curriculum renewal effort allowed the school to embark upon a redesign of the IPE learning domain to incorporate new core content from health systems science (HSS). We describe how our unique approach to IPE is being preserved as we innovate to produce graduates who are future thought leaders and "systems citizens," prepared to deliver patient care with an expanded knowledge of the health systems in which they will eventually practice.
\end{abstract}

Keywords Health systems science $\cdot$ Undergraduate medical education $\cdot$ Interprofessional education $\cdot$ Science of health care delivery $\cdot$ Systems citizens

\section{Background}

The Virginia Tech Carilion School of Medicine (VTCSOM) is a 4-year allopathic medical school that was founded in 2008 and graduated its first class in 2014. The medical school was launched as a result of a partnership between Virginia Tech, a

David W. Musick

dwmusick@vt.edu

1 Faculty Affairs, Virginia Tech Carilion School of Medicine, Roanoke, VA, USA

2 Interprofessionalism Learning Domain, Virginia Tech Carilion School of Medicine, Riverside Circle, Roanoke, VA 24016, USA

3 Academic Affairs, Virginia Tech Carilion School of Medicine, Roanoke, VA, USA

4 Office of Quality and Safety, Carilion Clinic, Roanoke, VA, USA

5 Community and Culture, Virginia Tech Carilion School of Medicine, Roanoke, VA, USA

6 School of Nursing, Radford University, Roanoke, VA, USA

7 Department of Physician Assistant Studies, Waldron College of Health and Human Services, Radford University, Roanoke, VA, USA

8 Obstetrics and Gynecology, Virginia Tech Carilion School of Medicine, Roanoke, VA, USA land grant public university, and Carilion Clinic, a private, notfor-profit health system. Its mission is "to develop physician thought leaders through inquiry, research and discovery, using an innovative curriculum based on adult learning methods in a patient centered context." The unique approach to medical education at this relatively new school has been described in detail elsewhere [1] and is built around four educational domains of learning: basic science, clinical science, research, and interprofessional education. Our curriculum is delivered in two phases, with phase 1 (years 1 and 2) divided into discreet blocks of time and requiring content from all four learning domains within each block of instruction and phase 2 (years 3 and 4 ) consisting of clinical, discipline-based educational rotations. The school uses a process of continuous review and educational quality improvement to periodically examine the overall curriculum, with a goal of ensuring that curricular content and delivery are pedagogically sound and "cutting edge."

Health systems science (HSS) has been described as the third pillar of medical education, being complementary to the traditional disciplines of basic and clinical science $[2,3]$. As healthcare delivery systems continue evolving the delivery of care to achieve the Quadruple Aim (i.e., improved patient experience of care, better care outcomes, lower costs, and clinician well-being), there is a corresponding need for medical education to develop and deliver content pertaining to how healthcare systems work and how the role of the clinical 
provider must include what has been described as systems citizenship. Currently, physicians may acquire skills in HSS during residency training as part of education pertaining to the general competency known as systems-based practice [4]. Subsequently, physicians may also encounter this content as part of ongoing professional development designed for career enhancement, with much of this learning taking place in their everyday workplace settings. Introducing HSS content early in the medical school curriculum is thought to contribute to learners' ability to work in teams, to enhance understanding of the larger healthcare delivery system, and to add actual value to care during their educational experiences in the clinical setting [5]. Currently, approximately 37 US medical schools are part of an American Medical Association consortium that, among many educational innovations, seeks to encourage US medical schools to include HSS content within their curricula [6]. Implementation of a robust HSS curriculum is critical to VTCSOM's mission of developing physician thought leaders.

A significant overarching component of HSS is interprofessional education (IPE). The VTCSOM has a decade-long track record of providing IPE, including emphasis on team science and the value of healthcare teams in care delivery $[7,8]$. From the inception of our medical school, we have delivered IPE content in collaboration with our partner institutions, Radford University Carilion (RUC, formerly the Jefferson College of Health Sciences) and Carilion Clinic. Our current curricular model features the requirement that medical, nursing, and physician assistant students spend significant time together in classroom, small group, and other settings learning "with, about and from each other to enable effective collaboration and improve health outcomes" [9]. IPE and HSS are conceptually synergistic and overlap in many areas, and the increasing emphasis on HSS presented our two schools with a unique opportunity to integrate new concepts of HSS and IPE within existing curricular time and in a manner that is inclusive of both educational partners. The dean of our medical school appointed a thirteen-member task force in August 2019 and charged the group with exploring how to create a robust experience for VTCSOM students in HSS, including an examination of what structures (academic departments, curricular domains) could best serve this purpose. Our intention was to create a forum to support co-creation of the HSS curriculum, which for us meant the active involvement of a variety of stakeholders. The task force was comprised of faculty from both phases of the curriculum and all four learning domains; clinicians who work with patient safety, quality of care, and simulation programs from our health system partner Carilion Clinic; and medical student representatives from years 1 through 3. RUC IPE course directors from the nursing and physician assistant programs provided input to the task force as well. We report here on the process and outcomes of the work of this task force, in hopes that the model developed will be useful to other schools who are contemplating the question of how to integrate IPE and HSS content into the curriculum.

\section{Task Force Workflow Process}

The task force on health systems science (HSS) held a series of nine 1-hour meetings beginning in August 2019 and ending in January 2020. Task force members conducted the majority of the work between meetings. This work included meeting with key stakeholders from the medical school as well as our partner educational institution (RUC) and our partner health system (Carilion Clinic); reviewing the existing literature on health systems science in medical education; reviewing several sets of educational competencies pertaining to HSS, IPE, and systems-based practice developed by various entities including the Association of American Medical Colleges (AAMC) and the Accreditation Council for Graduate Medical Education (ACGME); and reviewing content outlines of online modules pertaining to HSS developed by the American Medical Association (AMA) and the Institute for Healthcare Improvement (IHI). Task force meetings were well attended, and discussions of these materials and associated questions raised were robust and collegial.

As a substantive part of its work, the task force engaged with the VTCSOM Office of Academic Affairs to undertake a thorough review of the existing medical student curriculum, so that we would be better informed as to what HSS-related content was already represented in the medical student curriculum. We also reviewed content outlines from courses offered at RUC as part of their masters of science degree in nursing, which included content on HSS-related topics, with the possibility that some of the concepts covered in the masters level nursing course could be adapted for use in our revised curriculum which would be delivered to nursing students in an accelerated bachelor's degree program. Faculty colleagues from the RUC physician assistant training program provided similar information about where HSS concepts were being addressed in their courses. Because the medical school was the only entity that used a curriculum mapping software, our efforts at formal curriculum mapping were necessarily limited to the four domains in the medical student curriculum. For the medical school curriculum mapping exercise, we utilized a curriculum database system (One45) and based the search strategy on a review of HSS concepts found in the literature [10]. We used a total of 139 different search terms to identify and map required content from all four years and across all four curricular value domains (i.e., basic science, clinical science, research, and IPE). We gave particular attention to educational objectives from the existing IPE domain, core thirdyear clerkships (including clerkship passport requirements), problem-based learning cases from the first and second years, objectives from the first- and second-year longitudinal ambulatory clinical experience (LACE) program, and objectives from two required transition to residency courses for M4 students. The resulting search document was lengthy and displayed existing curricular content in two ways: categorized 
by the twelve major HSS content domains [10] and categorized chronologically by curriculum year and course. The results of the curriculum mapping project are displayed in Table 1, and this information was very helpful to the task force in terms of understanding where our curriculum is currently delivering HSS content, and in identifying current "gaps" in the curriculum that should be addressed.

Based on the information available and substantive discussions, we summarize specific recommendations made by the task force in the following sections organized by curriculum phase. Subsequent to the work of the task force, its recommendations were also discussed by the existing curriculum governance committees at our school. The overall plan was to roll out the HSS curriculum longitudinally, and therefore, the first impact was on the 130-140 students who were enrolled in August 2020 in the traditional IPE experiences (described below) which take place during the first year of the curriculum for medical students at the VTCSOM and for nursing and physician assistant students at RUC. The recommendations were reviewed and given final approval by the VTCSOM Medical Curriculum Committee. Subsequently, course design/implementation teams for both phases were constructed with oversight provided by the Office of Academic Affairs.

\section{Recommendations for Integrating HSS with IPE for the Pre-clinical Curriculum}

Maintain Variety of Content Delivery Methods The existing IPE curriculum most often features an approach that involves a combination of active didactic sessions followed by smallgroup learning wherein students are asked to apply the concepts learned in the immediately preceding presentation. For example, during the medical school's bioethics course, we typically have an opening presentation on one of the basic principles of clinical bioethics (e.g., autonomy, beneficence) with all students present, followed by students meeting in small groups to discuss the didactic material and related clinical cases. Similar small-group experiences involving medical, nursing, and physician assistant students take place throughout the year 1 IPE curriculum. The task force endorsed the content delivery methods featuring these combinations of active didactic sessions and small-group learning, feeling that this approach would be well-suited to the delivery of new HSS content. The task force also noted that this general approach is supplemented in other ways depending on the type of activity contemplated. For example, during our first-year IPE curriculum, we deliver a longitudinal patient case over three course sessions with the final session taking place in our simulation lab and featuring interactions with standardized patients as well as a simulated clinical care team meeting. Using a variety of content delivery methods, with emphasis on application to clinical care, was felt to be critically important to ensuring that new HSS content would be developmentally relevant to the students involved and maximize student learning.

In 2017, we modified the IPE domain in year 1 and created a two-track system whereby approximately half of the students participate in a culinary health track during half of the year, while the other half participate in a roles and scopes of practice track; they then switch tracks for the second half of the year. Students spent approximately one-half of the year in each of these two separate tracks. Students in the culinary health track participated in four related activities: 1) working in interdisciplinary teams in a local commercial kitchen to prepare and serve a meal to a designated group based on dietary needs; 2) completing an individual nutrition selfanalysis exercise; 3 ) working in interdisciplinary teams to

Table 1 Summary of existing VTCSOM objectives pertaining to health systems science

\begin{tabular}{lllll}
\hline HSS content domain* & Domain type & $\begin{array}{l}\text { Curriculum phase 1 } \\
\text { (years 1 and 2) }\end{array}$ & $\begin{array}{l}\text { Curriculum phase 2 } \\
\text { (years 3 and 4) }\end{array}$ & Total number of HSS-related objectives \\
\hline Evidence-based medicine/practice & Cross cutting & 34 & 17 & 51 \\
Professionalism and ethics & Cross cutting & 24 & 6 & 30 \\
Teamwork/IPE & Cross cutting & 26 & 41 & 67 \\
Scholarship & Cross cutting & 9 & 0 & 9 \\
Leadership/change agency & Cross cutting & 0 & 0 & 0 \\
Clinical informatics and HIT & Core & 8 & 3 & 11 \\
Healthcare policy/economics & Core & 18 & 21 & 39 \\
Healthcare structures/processes & Core & 15 & 14 & 29 \\
Health system improvement & Core & 9 & 1 & 10 \\
Population and public health & Core & 19 & 15 & 34 \\
Value-based care & Core & 1 & 3 & 4 \\
Total number of objectives identified & & 163 & 121 & 284 \\
\hline
\end{tabular}

*Based on Gonzalo J, Dekhtyar M, Starr SR, et al. Academic Medicine 92 (1): 123-131, 2017 
address clinical cases that featured nutrition and population health prominently; and 4) completing a community outreach service project related to nutrition. Students in the roles and scopes of practice track participated in a variety of activities including the following: 1) working through the aforementioned longitudinal patient case featuring teamwork and interdisciplinary care; 2) examining specific scopes of practice and related controversies pertaining to various health professionals; 3 ) studying team leadership in healthcare settings; 4) learning about the academic programs of study of their fellow students; and 5) considering how the "patient and caregiver voice" fits into the patient care team.

\section{Eliminate Low-Value Components of the Culinary Health} Track This recommendation to eliminate the commercial kitchen component was based on several factors including lack of consistent student buy-in, recommendations by our educational partners at RUC, challenging logistical requirements, and cost. However, it was recommended that the other components (community outreach focused on teaching clinical nutrition at an after school program in an underserved area of our city, classroom presentation on clinical nutrition, and student self-analysis of diet) be retained or absorbed into newly developed community service requirements for medical students. These changes allowed us to continue emphasizing HSS-related population health needs pertaining to clinical nutrition and to allow students to continue working in teams on clinical cases featuring the application of nutrition content to patient care.

\section{Streamline Content in the Roles and Scopes of Practice Track} This recommendation allowed for introduction of new material pertaining to team science, health policy, and orientation to a new patient navigation experience (to be developed, see subsequent section of this report). The streamlined roles and scopes of practice content, which retained most of the prior sessions described above and also consolidated some experiences, is currently being delivered during the medical school's first two instructional blocks of the academic year, which corresponds to the fall semester for RUC students. Participants in these first two blocks include first-year medical students and first-year physician assistant students and nursing students in an accelerated bachelor's degree program. Finally, new core content related to HSS will be delivered during instructional blocks 3 and 4 of the medical school's first academic year, which corresponds to the spring semester for RUC students. Participants in these two blocks will include first-year medical students and nursing students in the accelerated bachelor's degree program. A visual representation of the recommended year 1 curriculum is presented in Fig. 1.

Retain Real-time Observations of Nursing Practice This recommendation allows for the continuation of an IPE experience wherein second-year medical students apply IPE and HSS principles to the delivery of care in our health system. Medical students observe inpatient nurses in a variety of settings for approximately one half-day, learning valuable lessons about how nurses interface with physicians and other healthcare team members. These students also participate in an IPE simulation exercise involving an emergency room scenario designed to initially show dysfunction among team members, and consequently how an efficient team works together. Students are active participants in the simulation exercise, which focuses on running a code scenario. Both of these experiences ask medical students to participate in active learning and consider how they may apply their knowledge to help improve the situation from an IPE and HSS perspective.

Retain the Ethics Healthcare Team Challenge This recommendation preserved the major IPE activity involving medical, physician assistant, graduate nursing, and healthcare administration students working together in year 2. During the Legal and Bioethics block in year 2, medical students meet weekly to discuss bioethical principles following a presentation by a faculty member. The RUC students interact with each other online using the same bioethical resources (objectives, readings, cases). At the end of this 5-week period, we assemble all of the students for an afternoon, live and in real time, to discuss a complex patient case featuring ethical dilemmas that they have not encountered in the course. They are divided into IPE teams and debate and discuss the ethical principles of the case and prepare defenses of decisions that are made by the healthcare teams in the case. The Carilion Clinic Ethics Panel then facilitates a discussion with the students regarding their analysis and experiences with the case. This unique experience exemplifies how the cross-cutting HSS domain of bioethics can be delivered using IPE.

\section{Revise Didactic Learning Blocks for Second-Year Medical} Students These recommendations included re-structuring an existing block on narrative medicine, converting it from a fixed 6-week block of instruction to an alternate, to be determined longitudinal format, and expansion of a current 6-week block on public health, preventive medicine, population health, and health policy into two consecutive 6-week blocks, thus providing more in-depth coverage of core areas of HSS content.

\section{Develop a Value-added Experiential Learning Opportunity for} Second-Year Medical Students This new experience, such as a patient navigation program [11], will be created to begin with second-year medical students and continue into the third year. The plan is to implement this program with the Class of 2024 currently completing their first year of medical school. Potential collaborative partners for this experience will include our health system partner Carilion Clinic as well as 


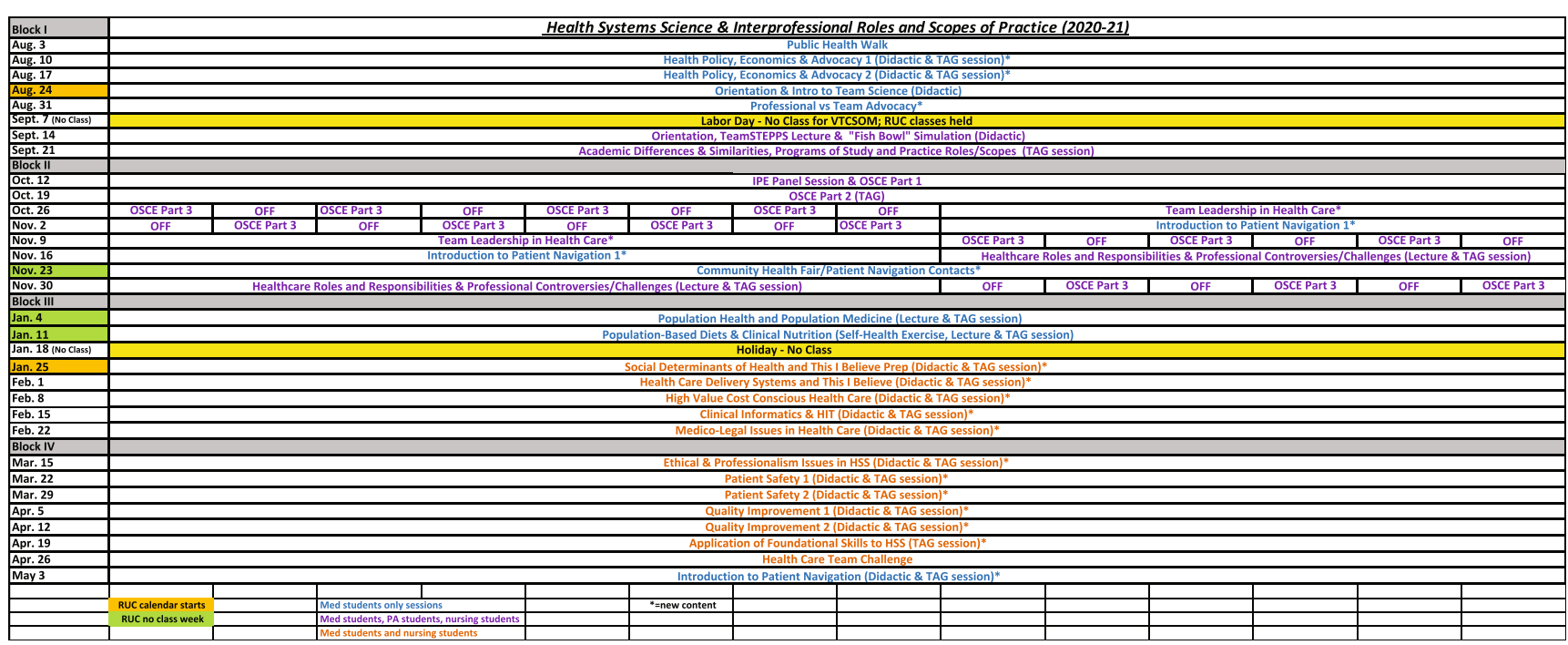

Fig. 1 Visual representation of the recommended year 1 curriculum

additional, to be identified community health agencies. To be of maximum impact, this experience must be designed to allow students to follow one or more patients/families longitudinally. Third-year core clerkship directors will be included in the planning of the experience. Related to this new experience, possible synergies with the existing VTCSOM Longitudinal Ambulatory Care Experience (LACE) program will be explored further. The LACE program is a community-based clinical skills learning experience required for first- and second-year medical students at the VTCSOM; it is a significant part of what would be considered a traditional "doctoring" course.

\section{Recommendations for Integrating HSS with IPE for the Clinical Curriculum}

While many medical schools deliver the majority of HSS content during the first two (primarily non-clinical) years of the curriculum, it is felt that the real "payoff" for this content occurs when students reach the clinical portion of their training and spend significant time interacting with patients as well as other members of the healthcare team. A substantive goal of HSS training is to provide students, residents, and/or practicing clinicians with information and strategies that will help them become "system citizens" and recognize the roles that physicians can play in improving the healthcare system itself [12]. Thus, incorporating HSS content into the clinical training context, and ensuring that its relevance is reinforced by clinical faculty, is critically important. The task force noted that the existing M3 clerkships currently have formal educational objectives related to interprofessional education and health systems science. This experience in delivery of IPE/ HSS content served to facilitate further thought about enhancement of HSS content in the clinical portion of the curriculum.

Recommendations for changes to the clinical portion of the VTCSOM curriculum included:

- Build upon the existing Domain Days that are already focused on aspects of HSS. These sessions, during the third year, are required and all third-year medical students, regardless of current clerkship rotation, come together for afternoon seminars on various topics. Currently, at least three of these sessions are focused on aspects of HSS including a surgical quality experience in a flipped classroom setting whereby students evaluate clinical cases from a quality of care perspective, an obstetrics and gynecology session focused on second victims and risk management, and an experiential mock "Disaster Day" simulation exercise involving medical students and IPE students from RUC. The entire Domain Day program will be integrated and refocused to achieve specific educational objectives related to HSS, with a majority of these sessions (currently numbering approximately 15 per year) featuring HSS content.

- Wherever possible, core knowledge content presented during years 1 and 2 of the curriculum should be reinforced in the clinical setting. This could be done via several methods (e.g., re-visiting problem-based learning cases from phase 1 , didactic presentations, journal club sessions, online modules offered by IHI, AMA, and/or others).

- HSS clinical "champions" in each clinical department represented by a core third-year clerkship will be identified and reimbursed for some portion of their time so that they can assist with implementation of clinically relevant HSS activities for students during the clinical years. These clinical champions will play a key role in facilitating the 
involvement of our students in meaningful and clinically experiential learning in tandem with our health system partner, Carilion Clinic.

- A robust elective experience (either 2 weeks or 4 weeks) in HSS will be developed for senior medical (fourth year) students. These electives will include topics found to be relevant in understanding and managing the COVID-19 pandemic of 2020.

\section{Assessment of Student Learning}

Measuring the impact of curricular changes, both on the individual student and the curriculum a whole, can be challenging. Much of the literature on assessment of student learning consists of Kirkpatrick level 1 or level 2 outcomes, such as learner reactions to the material and learner statements about the impact they felt the material will have on their future learning and/or practice [13]. Direct measurement of student knowledge about HSS will be facilitated by use of a new National Board of Medical Examiners (NBME) subject exam [14], as well as by other to be determined methods including assessment of HSS learning that will take place during the core clerkship year. The timing of the administration of the new HSS subject exam will be near the end of the fourth-year curriculum for medical students. However, since the major changes in IPE to HSS have occurred in our longitudinal roll-out in year 1, we administered the exam this past year to our M1 students who completed the traditional IPE curriculum. We will administer the exam to the current M1 class at the end of year 1 for comparison purposes. Other measures of student knowledge, attitudes, and competencies related to HSS are beginning to be developed but are still in their infancy due to the newness of HSS content within medical education. Curriculum leaders should engage very early in the curriculum development process with the school's office of student assessment and program evaluation, so that a detailed assessment and evaluation plan can be developed based on the educational objectives of each portion of the curriculum. When finalized, our overall assessment plan will ultimately be reflected in the course syllabi for each block of instruction, so that we can ensure that student learning is being assessed based on the educational objectives of the curriculum. And, the plan will also indicate how the impact of the recommended changes on the curriculum as a whole will be identified and monitored.

\section{Scholarly Opportunities}

Our medical students currently graduate with a Research in Translational Medicine Certificate following completion of specific coursework and a mentored research project. The project requirement is a hypothesis-driven study across any aspect of the translational science spectrum. We envision two ways in which the HSS curriculum can enhance the scholarly opportunities available to students once the full 4-year curriculum is implemented. A wider range of research opportunities will be available to students including hypothesisdriven projects focused on dissemination and implementation science or other aspects of HSS. We are also developing a HSS certificate program similar to our existing certificate for research in translational medicine. Medical students who have a strong interest in HSS will be encouraged to pursue additional learning beyond what is delivered in the required curriculum, resulting in receipt of the HSS certificate through designed electives and an additional project requirement. They may also receive a letter of distinction recognizing them for additional work (already part of the VTCSOM performance recognition system in other domains). Over time, we expect more of our students will be interested in pursuing joint $\mathrm{MD} / \mathrm{MPH}, \mathrm{MD} / \mathrm{MBA}$, and MD/MHA degrees.

The task force also recognized that with the introduction of significant curriculum change comes the opportunity to conduct education research related to the effectiveness of those changes. Therefore, faculty and/or professional staff interested in education research will be encouraged to conduct studies that will shed important light on the impact of curricular changes on student learning outcomes. And, faculty members experienced in conducting HSS-related research will be encouraged to provide VTCSOM students the opportunity to pursue hypothesis-driven research questions in order to satisfy the existing research requirements within the medical school curriculum.

\section{Faculty Development}

The development of a cadre of teaching faculty who can deliver HSS content in a variety of formats represents a formidable challenge. Some medical schools have established academy-type programs featuring in-depth faculty development experiences for faculty who wish to deliver various aspects of HSS content and can serve to accelerate the quality improvement and patient safety goals of their health system partners. At our academic health center, we have established the TEACH Academy (https://www.teach.vtc.vt.edu/) which provides faculty development opportunities for all faculty; this group is well poised to undertake a variety of approaches to developing faculty expertise in teaching HSS content. The TEACH program will develop a variety of activities designed to familiarize our faculty with HSS core content as well as teaching methods that can best be used to deliver the content. It will likely be necessary to identify a mechanism to provide protected time for faculty who participate in faculty 
development related to HSS. The task force felt that this work should be viewed as an urgent, high-priority goal for the medical school.

\section{Implementation Plans}

The task force noted that the curricular changes described would have an impact on several other issues. The first of these pertained to the name of the learning domain at the medical school, which has previously been identified as interprofessional education (IPE). At the VTCSOM, the curriculum is based on four foundational value domains of basic science, clinical science, research, and IPE. We are one of the few (and may be the only) medical schools to have an academic department of Interprofessionalism. Given that the construct of HSS encompasses content concerning IPE but is also broader in nature, it was recommended that the medical school administration consider re-naming the IPE learning domain to better reflect the revisions to the curriculum. Subsequently, the IPE domain name was changed to "Health Systems Science and Interprofessional Practice" (HSSIP). This change in nomenclature will solidify HSS content within the curriculum and may also help facilitate the selection and development of future faculty who have specific interest in HSS topics.

A second recommendation pertained to the curriculum map of HSS content. Once the final version of the new HSS portions of the curriculum has been officially approved and implemented across the entire curriculum, we will again produce a final and comprehensive curriculum mapping document that will allow interested parties to view the overall, "big picture" of where HSS content is being delivered across all four years of the medical school curriculum (to include both previous content and newly developed content). And, we will also establish a mechanism for identifying and labeling for student syllabi those parts of the curriculum that focus on IPE and HSS, whereby students will realize in "real time" that they are adding these new concepts to their accumulated knowledge and skill sets.

The final recommendation was to establish curriculum design/implementation teams that would build on the work of the task force. These teams of interprofessional experts have been formed and are currently designing the objectives, learning activities, and assessments necessary to fully integrate the HSS content into the entire 4-year curriculum, while at the same time enhancing existing successful IPE experiences. Curriculum leaders for each phase were identified who, working with the Senior Dean for Academic Affairs and under approval of the Medical Curriculum Committee, began overseeing the implementation process early in 2020 .

\section{Anticipatory Guidance in Times of Uncertainty}

The current pandemic presents sweeping challenges for curriculum delivery and may delay the implementation of new curricula at many medical schools. Counterbalancing these challenges is the highlighted relevance of HSS as a framework for understanding systemic factors that have promoted or impeded successful control of COVID-19 across the globe. We are fortunate that our HSS curriculum is situated within established curricular domains and does not require a larger scale transformation of our curriculum block design and learning assessments.

When the COVID-19 pandemic happened in March 2020, we quickly realized the need to move instruction online during the spring and summer months, so the implementation of the new HSSIP curriculum changed accordingly. The concerns of trying to have students learn in a virtual environment were assessed for feasibility. The attempt to have students in their face to face small learning teams of $8-10$ was eventually thwarted when it was determined that not enough of the right-sized rooms existed for the students to socially distance together. When it became inevitable that the majority of the new HSSIP curriculum for the fall 2020 semester needed to be delivered virtually, work was undertaken to develop both large- and small-group online sessions to be as friendly and interactive as possible. As of this writing, our HSS curriculum delivery relies on a very limited number of in-person smallgroup sessions and a mixture of small- and large-group virtual sessions. As our first-year students gain experience with the small-group process, we are developing greater confidence that a required transition to a fully virtual HSS curriculum would be successful if circumstances required it. As the first year of the new HSSIP curriculum unfolds, new challenges will surely arise, but with the curriculum foundation in place through careful and thoughtful planning, it is felt that any barriers can be overcome effectively. Later years of HSS implementation will involve more patient contact in office and hospital settings. Our clinical preceptors and health system partners accepted the return of our students to the clinical environment starting in July of 2020. We are all looking forward to implementing the new features of HSS planned for future years.

\section{Conclusions}

Health systems science can enhance future physicians' understanding of the microsystems in which they work, and of higher level forces that drive the quality and value of healthcare. As the COVID-19 pandemic demonstrates, the health of patients and populations relies on systems that are not immediately relevant in everyday clinical practice, but which the public expects to function successfully in critical 
times. Despite its importance, achieving competence in HSS may seem challenging in a curriculum with little to no room for additional content.

We describe a collaborative and systematic process for substantial curricular change that preserves the unique strengths of a medical school's IPE domain while introducing mission critical HSS content. This curricular renewal is designed to activate and enhance the value of the clinical learning environment by using a variety of content delivery methods to ground first-year students in HSS content and introduce value-added experiential HSS in the second curricular year. Our renewal plan will also showcase HSS principles in action during the third-year curriculum and offer additional HSS educational experiences for senior medical students who wish to pursue HSS elective experiences during their final year of medical school. Assessments of student learning will drive continued improvements and refinements in the curriculum. Certificate and master's programs will allow interested students to deepen their scholarly preparation in the aspects of HSS that interest them most. The goal of our curriculum renewal process is to engage our students in new ways around important HSS content and competencies, so they will ultimately become systems citizens that provide outstanding patient care.

Authors' Contributions All authors contributed to the study conception and design. The first draft of the manuscript was written by David W. Musick and all authors commented on previous versions of the manuscript. All authors read and approved the final manuscript. All authors whose names appear on the submission:

1. Made substantial contributions to the conception or design of the work, or the acquisition, analysis, or interpretation of data;

2. Drafted the work or revised it critically for important intellectual content;

3. Approved the version to be published; and

4. Agree to be accountable for all aspects of the work in ensuring that questions related to the accuracy or integrity of any part of the work are appropriately investigated and resolved.

Data Availability Not applicable

\section{Compliance with Ethical Standards}

Conflict of Interest The authors declare that they have no conflicts of interest.

Code Availability Not applicable

\section{References}

1. LaConte L, Johnson TA, Vari RC, Friedlander MJ. Research immersion at the Virginia Tech Carilion School of Medicine - an integrated curriculum Producing Scientist Physicians for the future of healthcare. Med Sci Educ. 2013;23(1S):122-34.

2. Lucey CR. Medical education: part of the problem and part of the solution. JAMA Intern Med. 2013;173(17):1639-43.

3. Gonzalo JD, Haidet P, Papp KK, Wolpaw DR, Moser E, Wittenstein RD, et al. Educating for the 21st-century health care system: an interdependent framework of basic, clinical, and systems sciences. Acad Med. 2017;92(1):35-9.

4. Pruitt Z, Mhaskar R, Kane BG, Barraco RD, et al. Development of a health care systems curriculum. Adv Med Educ Pract. 2017. Accessed online March 22, 2020;8:745-53. https://doi.org/10. 2147/AMEP.S146670.

5. Gonzalo JD, Davis C, Thompson BM, Haidet P. Unpacking medical students' mixed engagement in health systems science education. Teach Learn Med. 2019;26:1-9.

6. American Medical Association. Creating a community of innovation. Chicago: American Medical Association; 2017.

7. Vari PM, Lash J, Brown SS, Porter AG, Trinkle D, Garber JS, et al. Collaborative practice education: the effect of an interprofessional teamwork course on students' knowledge and skills. Med Sci Educ. 2013;23(3S):494-501.

8. Trinkle DB, Schnurman-Crooke A, Porter AG, Vari PM, et al. Stumbling and succeeding together: a new medical school's collaborative initiative to elevate interprofessionalism as a signature hallmark in training future healthcare leaders. Med Sci Educ. 2014;24: 219-23.

9. World Health Organization (WHO). Framework for action on interprofessional education and collaborative practice. Geneva: WHO; 2010. Available online at https://www.who.int/hrh/ resources/framework_action/en/. Accessed March 22, 2020

10. Gonzalo JD, Dekhtyar M, Starr SR, Borkan J, Brunett P, Fancher T, et al. Health systems science curricula in undergraduate medical education: identifying and defining a potential curricular framework. Acad Med. 2017;92(1):123-31.

11. Gonzalo JD, Lucey C, Wolpaw T, Chang A. Value-added clinical systems learning roles for medical students that transform education and health: a guide for building partnerships between medical schools and health systems. Acad Med. 2017;92(5):602-7.

12. Davis CR, Gonzalo JD. How medical schools can promote community collaboration through health systems science education. AMA J Ethics. 2019;21(3):E239-47.

13. Kirkpatrick DL. Techniques for evaluating training programs. J Am Soc Train Direct. 1959;13(3):21-6.

14. New health systems science exam now available to all medical schools. National Board of Medical Examiners: August 29, 2019. Reviewed online March 22, 2020, available online at https://www. nbme.org/news/new-health-systems-science-hss-examinationnow-available-all-medical-schools.

Publisher's Note Springer Nature remains neutral with regard to jurisdictional claims in published maps and institutional affiliations. 